\section{Technique of retinal gene therapy: delivery of viral vector into the subretinal space}

K Xue, M Groppe, AP Salvetti and RE MacLaren
Nuffield Laboratory of Ophthalmology, University of Oxford \& Oxford Eye Hospital, Oxford University Hospitals NHS Foundation Trust, Oxford, UK

Correspondence: RE MacLaren, Nuffield Laboratory of Ophthalmology, University of Oxford \& Oxford Eye Hospital, Oxford University Hospitals NHS Foundation Trust, Level 6 West Wing, John Radcliffe Hospital, Headley Way, Oxford OX3 9DU, UK

Tel: +44(0) 1865741166;

Fax: +44(0) 1865231534 .

E-mail: enquiries@ndcn.ox. ac.uk

Received: 31 December 2016

Accepted in revised form: 3 July 2017

Published online:

18 August 2017

\begin{abstract}
Purpose Safe and reproducible delivery of gene therapy vector into the subretinal space is essential for successful targeting of the retinal pigment epithelium (RPE) and photoreceptors. The success of surgery is critical for the clinical efficacy of retinal gene therapy. Iatrogenic detachment of the degenerate (often adherent) retina in patients with hereditary retinal degenerations and small volume (eg, $0.1 \mathrm{ml}$ ) subretinal injections pose new surgical challenges.

Methods Our subretinal gene therapy technique involved pre-operative planning with optical coherence tomography (OCT) and autofluorescence (AF) imaging, $23 \mathrm{G}$ pars plana vitrectomy, internal limiting membrane staining with Membrane Blue Dual (DORC BV, Zuidland, Netherlands), a two-step subretinal injection using a $41 \mathrm{G}$ Teflon tipped cannula (DORC) first with normal saline to create a parafoveal bleb followed by slow infusion of viral vector via the same self-sealing retinotomy. Surgical precision was further enhanced by intraoperative OCT (Zeiss Rescan 7000, Carl Zeiss Meditec AG, Jena, Germany). Foveal functional and structural recovery was evaluated using bestcorrected Early Treatment Diabetic Retinopathy Study (ETDRS) visual acuity, microperimetry and OCT.

Results Two patients with choroideremia aged 29 (P1) and 27 (P2) years, who had normal and symmetrical levels of best-corrected visual acuity (BCVA) in both eyes, underwent unilateral gene therapy with the fellow eye acting as internal control. The surgeries were uncomplicated in both cases with successful detachment of the macula by subretinal vector injection. Both treated eyes showed recovery of BCVA (P1: 76-77 letters; P2: 84-88 letters) and mean threshold sensitivity of the central macula (P1: 10.7-10.7 dB; P2: 14.2-14.1 dB) to baseline within a month. This was
\end{abstract}

accompanied by normalisation of central retinal thickness on OCT.

Conclusions Herein we describe a reliable technique for subretinal gene therapy, which is currently used in clinical trials to treat choroideremia using an adeno-associated viral (AAV) vector encoding the $\mathrm{CHM}$ gene.

Strategies to minimise potential complications, such as avoidance of excessive retinal stretch, air bubbles within the injection system, reflux of viral vector and post-operative vitritis are discussed. Eye (2017) 31, 1308-1316; doi:10.1038/eye.2017.158; published online 18 August 2017

\section{Introduction}

Retinal gene therapy offers our best hope for treating inherited retinal dystrophies in the foreseeable future based on successful proofof-principle in animal models and promising data from human clinical trials. Adenoassociated viral (AAV) vector based gene therapy for RPE65-associated Leber congenital amaurosis (LCA) and choroideremia (CHM) have entered Phase III and II clinical trials respectively. In both cases, demonstrating long-term improvements in visual function in those patients in whom early treatment effects were observed. ${ }^{1-4} \mathrm{AAV}$ gene therapies for other monogenic retinal disorders are under investigation, eg MERTK-associated retinitis pigmentosa, ${ }^{5} \mathrm{X}$-linked retinoschisis (XLRS), CNGA3-associated achromatopsia and ND4-associated Leber hereditary optic neuropathy (LHON). ${ }^{6}$ In addition, gene therapy has been used to create sustained ectopic production of a secreted anti-vascular endothelial growth factor protein as an alternative way of treating wet age-related macular degeneration. ${ }^{7}$

Unlike conventional drug treatments, retinal gene therapy is a complex biological treatment 
whose efficacy could depend on a multitude of factors, including optimised AAV vector design, high quality vector production and intervention at an appropriate stage of the disease. ${ }^{8}$ The least unpredictable of all the variables is the surgical delivery to the subretinal space, which must minimise trauma but maximise viral transduction of the target cells. Reflux of the vector suspension into the vitreous cavity will, by definition, reduce the subretinal dose applied to the target cells. Furthermore, intravitreal AAV particles may stimulate an immune response to the viral capsids (vitritis), which may further degrade the number of effective AAV particles in the subretinal space. Although intravitreal injection of vector can be used to treat diseases primarily affecting the inner retina (eg, Müller cells in XLRS and ganglion cells in LHON), it is disadvantaged by vector dilution within the vitreous cavity. A modification of this approach by injecting under the internal limiting membrane (ILM) may avoid some of these problems. ${ }^{9}$ Here we focus on the technique of subretinal gene therapy, which offers a targeted approach to treating outer retinal degenerations caused by genetic mutations in the retinal pigment epithelium (RPE) and photoreceptors. Administration of AAV vector into the potential space between the photoreceptor outer segments and the RPE allows treatment of a specific area of the retina (eg, the macula), generates a high local concentration of vector to enhance the proportion of cells transduced, and helps to evade adaptive immune responses. Furthermore, the hydrostatic force of the subretinal injection will pump vector suspension into the extracellular space of the outer retina, thereby providing a potential reservoir of AAV particles for further transduction after the initial subretinal fluid has been reabsorbed.

\section{Materials and methods}

\section{Pre-operative assessment}

As part of surgical planning prior to undergoing gene therapy, choroideremia patients with confirmed genetic mutations in the CHM gene were assessed with a range of baseline visual function tests as well as retinal imaging. These included best-corrected visual acuity (BCVA) using the ETDRS chart at $4 \mathrm{~m}$, MAIA microperimetry (Centervue, Padova, Italy) using the 10-2 pattern and 4-2 dB bracketing strategy under mesopic condition (background luminance $1.3 \mathrm{~cd} / \mathrm{m}^{2}$ ), spectral domain OCT (Spectralis, Heidelberg Engineering, Heidelberg, Germany) and fundus autofluorescence (AF, BluePeak, Spectralis). ${ }^{10}$

\section{AAV vector}

The AAV vector for choroideremia gene therapy consists of a chicken $\beta$-actin (CBA) promoter, human $C H M$ cDNA encoding Rab-escort protein 1 (REP1), bovine poly(A) signal and a modified Woodchuck hepatitis virus posttranscriptional regulatory element (WPRE), packaged in the AAV serotype 2 capsid, as described previously. 3,10 The subretinal dose of AAV.REP1 vector was $0.1 \mathrm{ml}$ of a $10^{12}$ genome particles ( $\mathrm{gp}$ ) per $\mathrm{ml}$ solution containing $0.001 \%$ PF-68, a surfactant shown to reduce adhesion of AAV viral capsids to the materials of the injection system. $^{11}$

\section{Subretinal injection}

The technique of subretinal delivery of AAV vector described below is currently used in two interventional clinical trials of gene therapy for choroideremia: (1) An open label dose escalation Phase 1 clinical trial of retinal gene therapy for choroideremia using an adenoassociated viral vector encoding Rab-escort protein 1 (ClinicalTrials.gov identifier: NCT01461213), and (2) An open label Phase 2 clinical trial of retinal gene therapy for choroideremia using an adeno-associated viral vector encoding REP1 (ClinicalTrials.gov identifier: NCT02407678).

As part of the trial protocol, peri-operative corticosteroid (oral prednisolone) was given, starting two days prior to surgery at $1 \mathrm{mg} / \mathrm{kg}$ for 10 days, then $0.5 \mathrm{mg} / \mathrm{kg}$ for 7 days, $0.25 \mathrm{mg} / \mathrm{kg}$ for 2 days and then $0.125 \mathrm{mg} / \mathrm{kg}$ for 2 days. For an $80 \mathrm{~kg}$ man, the doses per day would be equivalent to $80,40,20$ and $10 \mathrm{mg}$. Gastric protection was given in the form of oral omeprazole $20 \mathrm{mg}$ twice daily for the duration of the corticosteroid regime.

The surgery consisted of a standard three-port 23-gauge pars plana vitrectomy, induction of posterior vitreous detachment (if not already present), core and peripheral vitrectomy, and ILM staining with Membrane Blue Dual (DORC, Zuidland, The Netherlands). Macula detachment was induced by subretinal injection of a balanced salt solution (BSS). The injection device was an extendible $41 \mathrm{G}$ polytetrafluoroethylene (Teflon) blunttipped subretinal cannula (DORC) connected to the viscous fluid control (VFC) port of the Alcon Constellation Vision System (Alcon, Fort Worth, TX, USA). The infusion pressure was controlled via a footpedal with the maximum limit set to the minimum that would produce a continuous flow of fluid rather than a stream of droplets-generally this was 12-16 psi.

After flushing and testing for bubbles by immersing the tip of the cannula into a BSS dish outside of the eye, the needle was retracted and introduced through one of the 

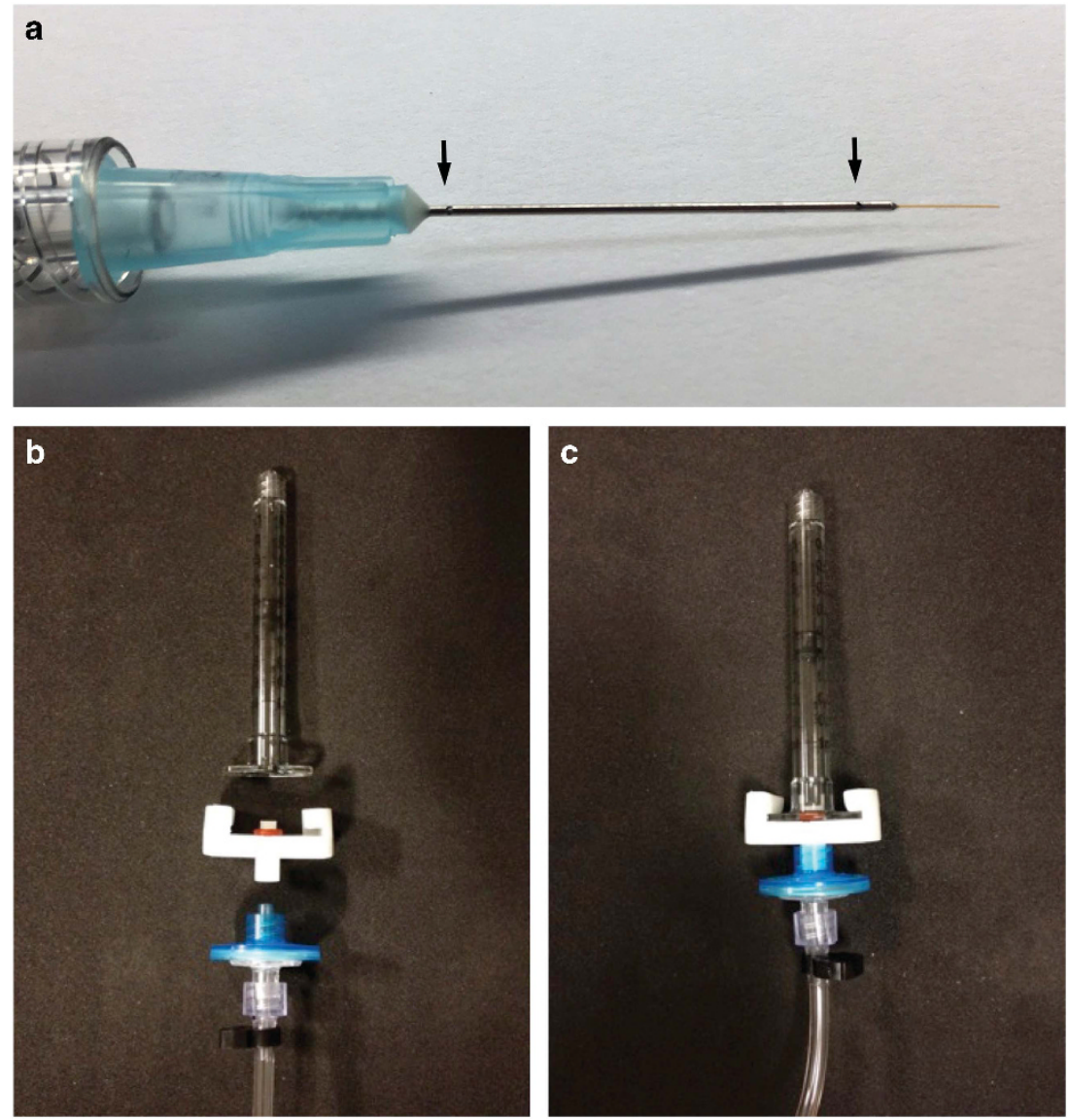

Figure 1 Subretinal gene therapy injection system. (a) A dual-bore $41 \mathrm{G}$ blunt polytetrafluoroethylene (Teflon) tipped cannula mounted within a $23 \mathrm{G}$ steel shaft (DORC, Zuidland, The Netherlands). Arrows indicate proximal and distal pressure relief holes along the shaft, which allow evacuation of excess vitreous fluid out of the eye during the injection. (b) Components of the subretinal injection system consisting of a $1 \mathrm{ml}$ BD syringe, custom syringe lock with a rubber O-ring, air filter and polyethylene connection tubing (Alchimia, Ponte S Nicolò, Italy). (c) Assembled injection system.

ports. The needle tip was then advanced and guided to the retina in the region of the superior vascular arcades, but avoiding the blood vessels, where the subretinal injection was initiated. Once propagation of the subretinal BSS bleb towards the fovea was seen and confirmed by intraoperative OCT (Zeiss Rescan 7000, Carl Zeiss Meditec AG, Jena, Germany) displayed within the surgeon's microscope eyepiece as overlay, the BSS needle was retracted.

Next $0.3 \mathrm{ml}$ of AAV.REP1 vector was loaded into a $1 \mathrm{ml}$ syringe (Beckton Dickinson, Oxford, UK), fitted with a disposable dual-bore $41 \mathrm{G} / 23 \mathrm{G}$ Teflon tipped cannula (DORC) and connected to the Constellation system via a custom syringe lock and standard VFC tubing (Figure 1). Care was taken during BSS and vector loading to purge all air bubbles from the injection systems as described previously. ${ }^{11}$ As with the initial BSS injection, the primed vector injection system was first tested in a pot of saline under the microscope prior to entering the eye. A maximum dose of $0.1 \mathrm{ml}$ of the AAV vector was slowly infused into the subretinal bleb through the existing retinotomy under foot-pedal control. This slow approach avoided over-stretch of the retina because there is often insufficient subretinal volume for $100 \mu \mathrm{l}$ of fluid in the endstage CHM patients. Occasionally an additional retinotomy was made with the $41 \mathrm{G}$ cannula to fully detach a large 'retinal island' or treat a non-contiguous retinal island as seen on AF imaging. The progression of retinal detachment, including detachment of the fovea, was carefully monitored by intraoperative OCT. In some cases where there were areas of focal thinning, heavy liquid was used to provide internal tamponade of the retina, similar to the technique described by Maguire to protect the thin fovea in LCA patients. ${ }^{8}$ After delivery of vector into the subretinal space, the vitreous cavity was irrigated (to clear any refluxed free floating AAV particles). In most cases the eye was left fluid filled (to minimise vector reflux and risk of cataract formation) and all sclerostomies were sutured with 8-0 polyglactin (Vicryl, Ethicon, Johnson and Johnson, New Brunswick, NJ, USA). 


\section{Results}

The typical surgical technique for subretinal gene therapy and perioperative findings are illustrated using data and images from two recent CHM patients (both brothers) aged 29 (Patient 1) and 27 (Patient 2) years. Both underwent unilateral gene therapy on the same day as part of a Phase II clinical trial, with the fellow eye acting as an internal control. The patients had normal and symmetrical levels of BCVA in both eyes at baseline-76 letters OD (treated) versus 79 OS (control) in P1, and 87 OD (control) versus 84 OS (treated) in P2 (Supplementary Table I) - but with significant loss of peripheral visual fields as demonstrated by microperimetry. The surgeries were uncomplicated in both cases with successful detachment of the residual central retinal 'island' by subretinal vector injection. Intraoperative OCT enabled early detection of the build-up of subretinal fluid at the injection site during bleb initiation (while excluding suprachoroidal injection), and provided early indication of the direction of propagation of the bleb, thus allowing the surgeon to adjust the position of the cannula as required. It also helped to confirm foveal detachment in both cases, indicating that the retinal structure responsible for visual acuity was targeted successfully (Figure 2).

The preoperative baseline assessments used for surgical planning were BCVA, MAIA microperimetry, OCT and AF imaging. These helped to determine the functional and structural integrity of the fovea, and the area of preserved photoreceptors (ellipsoid zone on OCT) and RPE cells (AF 'islands') to which gene therapy could be applied, since the AAV vector is designed to replace the non-functioning CHM gene within surviving cells but would not be expected to rescue areas where the cells have already been lost. ${ }^{12}$ OCT was also used to rule out cystoid macular oedema, retinoschisis or choroidal neovascularisation at baseline, which could occasionally associate with CHM. The 10-2 MAIA microperimetry assessed the threshold sensitivities of the central $20^{\circ}$ field (covered by 68 points) with 'Stable' fixation
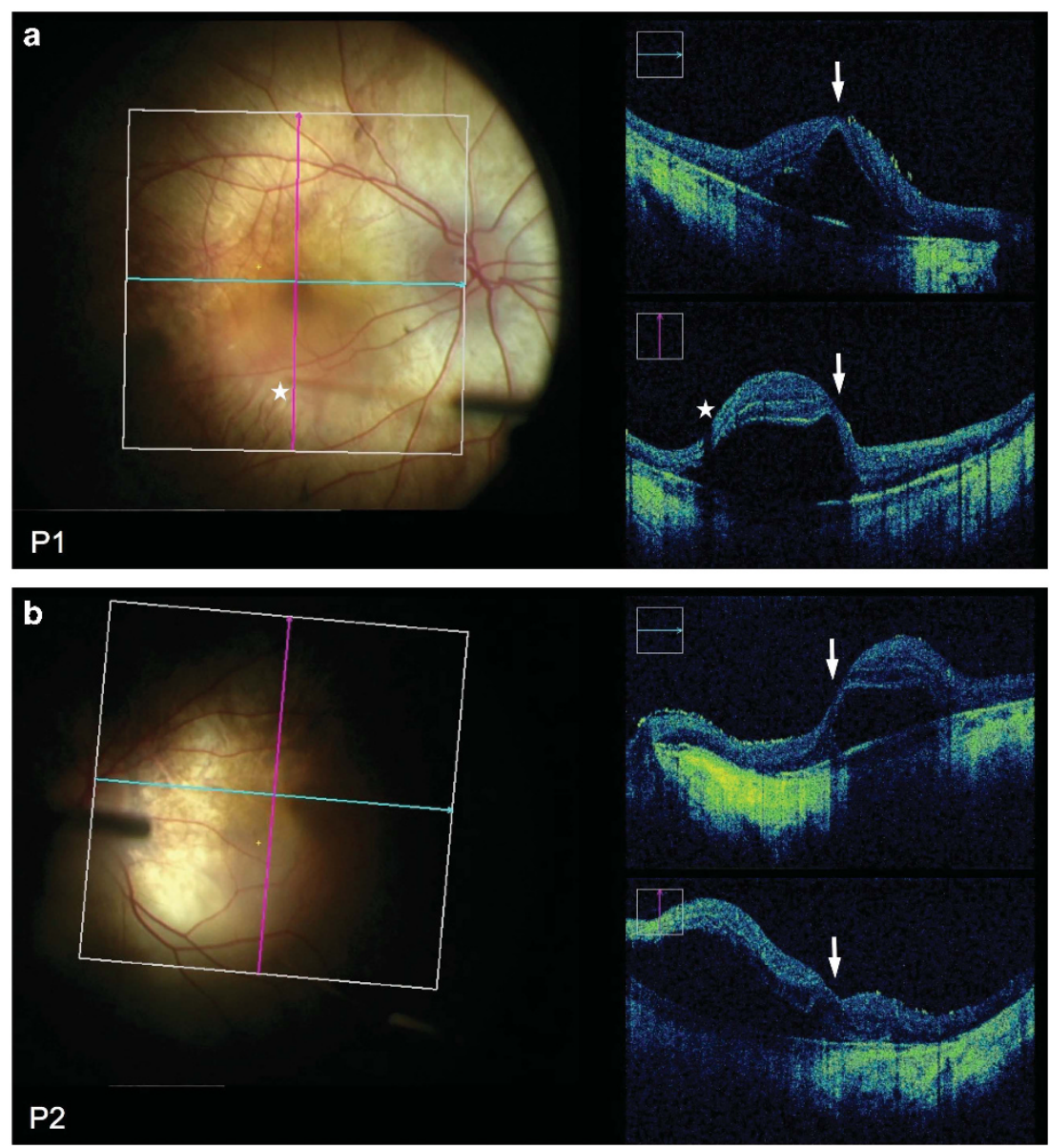

Figure 2 OCT-guided subretinal injection. Microscope photograph and simultaneous intraoperative OCT (Zeiss Rescan 7000, Carl Zeiss Meditec AG, Jena, Germany) during subretinal gene therapy for choroideremia in Patient 1 OD (a) and Patient 2 OS (b). Blue and pink grid lines indicate the locations of horizontal and vertical OCT B-scans respectively. The fovea could be seen to be detached in both cases (arrows). Stars indicate the tip of the subretinal cannula (seen out of focus) and the corresponding shadow cast on the OCT. 
stability achieved in all cases (Figure 3). The areas with detectable threshold sensitivities $(>2.0 \mathrm{~dB}$, coloured dots) plotted over the inbuilt confocal scanning laser ophthalmoscope (cSLO) image could be seen to correspond to the area of residual autofluorescence, whereas areas with non-detectable sensitivities $(<2.0 \mathrm{~dB}$, black dots) corresponded to atrophic regions lacking autofluorescence. The fixation points (shown by cluster of fine green dots on the microperimetry maps) corresponded to the areas with the greatest threshold sensitivities (up to $16.0 \mathrm{~dB}$ ) and the location of the foveal dip on the OCT.

Post-operatively, the retinotomies self-sealed without sequelae and subretinal fluid resolved within 24 hours (Figure 4). Structural recovery of the retina following iatrogenic detachment of the macula generally occurred within one month (Figure 4 and Supplementary Table S1). In both patients, the mean central retinal thickness (of the $1 \mathrm{~mm}^{2}$ ETDRS circle) and retinal volume (from the ILM to the Bruch's membrane within the $1 \mathrm{~mm}^{2}$ ETDRS circle) were increased from baseline on day 1, partially resolved by 1 week and returned to baseline by 1 month postoperatively. Since the retinal oedema was induced by the positive pressure subretinal injection (as seen with the intraoperative OCT imaging), its persistence on postoperative day 1 indicates that intra-retinal fluid persists for longer than subretinal fluid. This may have additional advantages for retinal transduction, particularly for photoreceptors, as vector particles will be surrounding and in direct contact with outer nuclear layer cells long after the subretinal fluid has been reabsorbed by the RPE. This is a potential advantage in using the subretinal compared with intravitreal approach. ${ }^{13}$ The resolution of retinal oedema by 1 month following gene therapy was associated with preservation of the 'retinal islands' on AF imaging (Figure 3). Functional recovery was demonstrated by return of BCVA to baseline levels in both patients by 1 month follow-up-77 letters OD (treated) versus 83 OS (control) in P1, and 87 OD (control) versus 88 OS (treated) in P2. In addition, the mean central retinal sensitivities of the treated eyes on microperimetry also returned to baseline at 1 month.

\section{Discussion}

While many early gene therapy clinical trials have recruited patients with advanced disease, the reassuring safety and efficacy data emerging from these trials would suggest that early intervention might be more beneficial to preserve retinal cells prior to a potentially irreversible stage of structural disorganisation. In choroideremia, we have previously shown that the edge of photoreceptor loss as seen on OCT closely follows the extent of RPE loss on AF image, suggestive of mutually dependent cell a

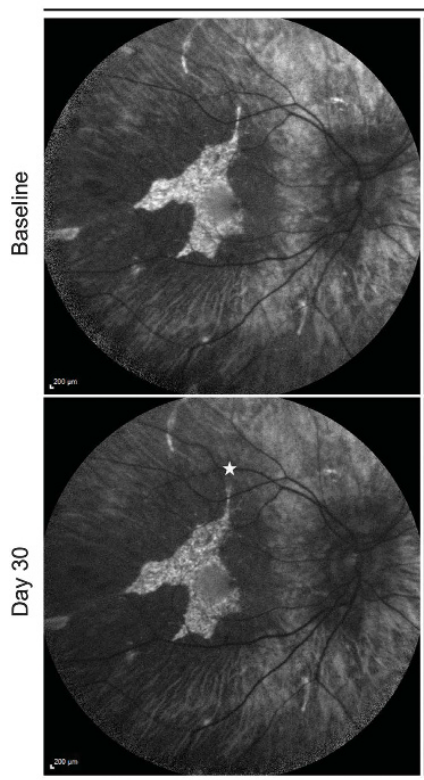

P1

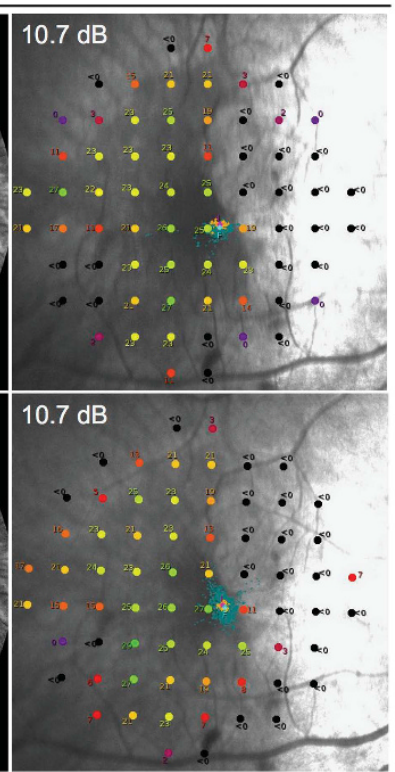

b

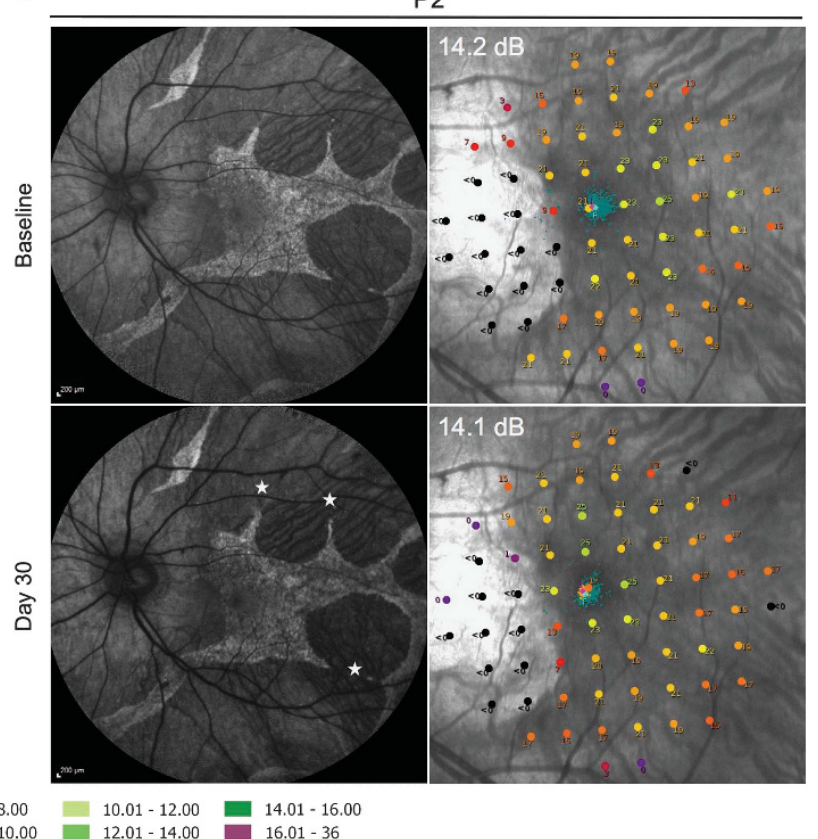

Figure 3 Preservation of autofluorescence (AF) island and central retinal sensitivity following subretinal gene therapy. Baseline and 30 days post-operative AF images and 10-2 MAIA microperimetry of the treated eye of Patient 1 (a) and Patient 2 (b) are shown. Stars indicate the locations of retinotomies (one in P1 and three in P2) performed during the subretinal BSS and vector injections, which successfully detached the central retinal island in each case. The threshold retinal sensitivities of the central $20^{\circ}$ of the macula were represented by 68 colour-coded points superimposed on the cSLO image. The points of fixation are shown as clusters of fine green dots. 
a
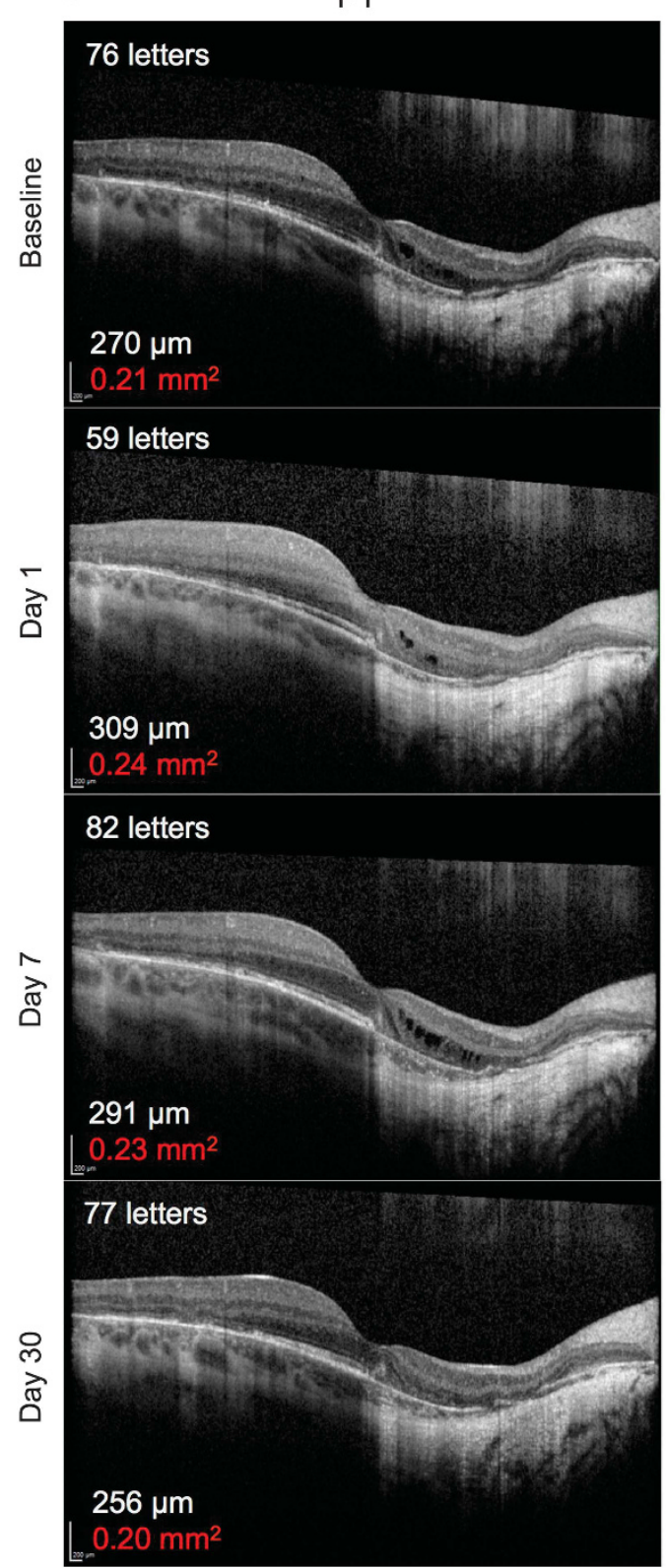

b
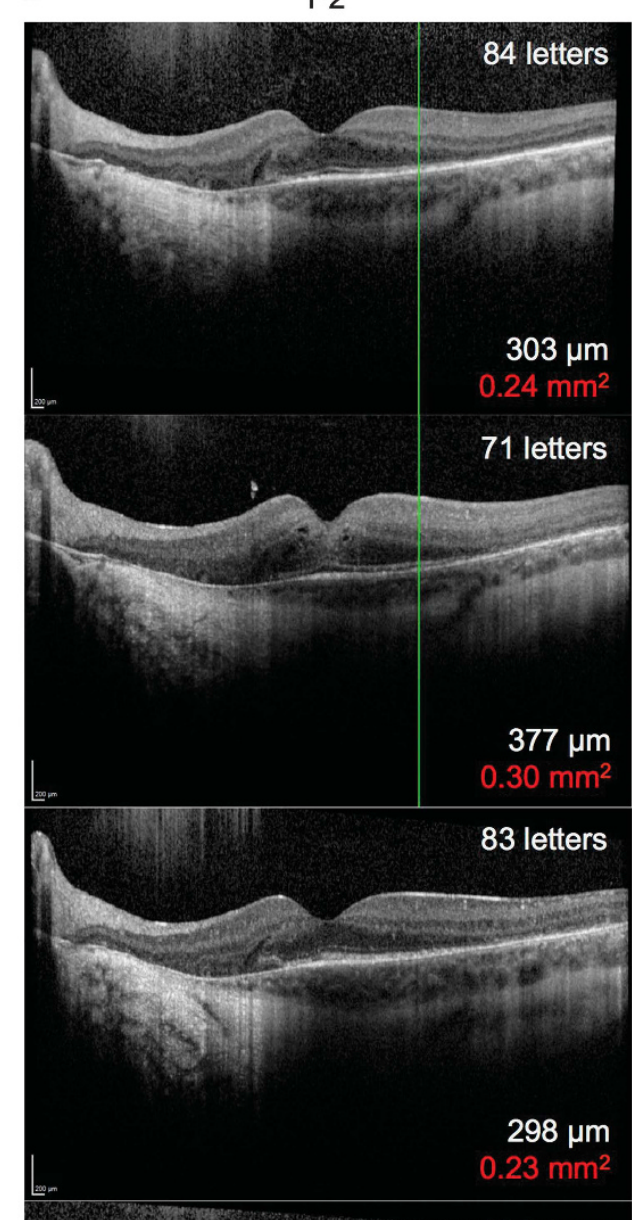

88 letters

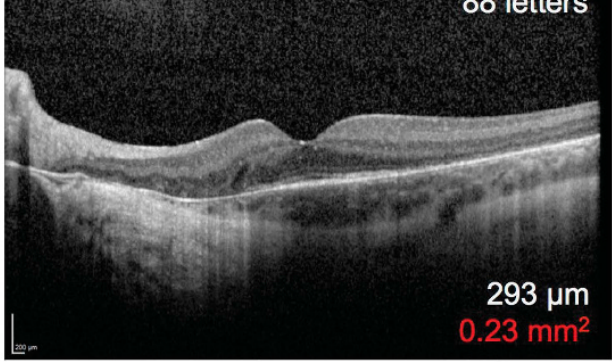

Figure 4 Resolution of subretinal fluid and recovery of visual acuity following subretinal gene therapy. Baseline and post-operative day 1, 7 and 30 foveal OCT images from Patient 1 (a) and Patient 2 (b) as described in Figures 2 and 3. The best-correct visual acuity (number of ETDRS letters) at each time-point is shown in the top corner of each OCT image. The mean central retinal thickness of the $1 \mathrm{~mm}^{2}$ ETDRS circle (in $\mu \mathrm{m}$ ) and retinal volume from the ILM to the Bruch's membrane within the $1 \mathrm{~mm}^{2}$ ETDRS circle (in mm², red) are shown in the bottom corner of each OCT image. No significant changes in mean central retinal thickness or volume were observed in the fellow eye of either patient (Supplementary Table I).

survival, although some photoreceptors appeared to persist beyond the edge of RPE loss in the form of outer retinal tubulations. ${ }^{14}$ Moreover, our natural history study showed the mean lifetime rate of shrinkage of the AF area in $\mathrm{CHM}$ to be $7.7 \%$ per year, meaning that the absolute area of retina lost per year is greater at younger age. ${ }^{15}$ Prior to subretinal gene therapy, combined OCT and AF imaging are essential for confirming retinal structural integrity within the treatment zone to tolerate a safe subretinal approach. It is important to identify areas of excessive retinal thinning, which could open up under subretinal pressure to form a secondary retinal hole, leading to failure of BSS/vector bleb propagation, egress of vector and potentially reduced visual acuity. A bubble of heavy liquid may be placed over an area of excessive retinal thinning during the subretinal injection to help 
prevent the formation of a secondary retinal hole. Microperimetry and OCT are also particularly informative for identifying eyes in which the foveal photoreceptors lie immediately on the advancing 'frontier' of RPE loss since these might be particularly susceptible to visual acuity fluctuations following an iatrogenic retinal detachment. It remains unclear whether the cells around the edge of the residual AF islands are amenable to rescue by gene supplementation. Continued degeneration of these 'border cells' could potentially lead to a paradoxical drop in vision in the short-term but slowing of disease progression in the long-term.

The surgical technique of subretinal gene therapy builds upon established subretinal procedures such as subretinal tissue plasminogen activator (tPA) injection and macular translocation surgery. However, specific considerations must be made to adapt the technique to the retinal characteristics in hereditary retinal dystrophies. Unlike subretinal tPA injection where the subretinal space has already been opened up and demarcated by blood, inducing a clean foveal detachment in choroideremia is unpredictable due to the subretinal adhesions surrounding the AF islands which strongly resist extension of the iatrogenic detachment. Also unlike subretinal tPA patients who have underlying macular pathology, choroideremia patients undergoing gene therapy often have normal BCVA (6/6), which raises new challenges to minimise foveal stretch during vector delivery. Depending on the approximate diameter of the AF island in each eye, the maximum volume $\left(V_{\max }\right)$ of the subretinal bleb could be approximated using the spherical cap formula (Figure 5) ${ }^{16}$ - an injection volume beyond $V_{\max }$ would be expected to cause either retinal stretch (with resulting mechanical disruption of nerve fibres and neuronal synapses) and/or reflux from the retinotomy Knowing $V_{\max }$ would allow the surgeon to predict the maximum dose for a safe subretinal injection. It would also inform clinical trial design since it may not be practical to define a standard dose volume for all choroideremia patients, but rather tailor the dose to the AF area being treated.

The incorporation of foot-pedal control and intraoperative OCT helps to minimise excessive retinal stretch by allowing the surgeon to monitor in real-time the amount of the retinal elevation induced by the BSS and vector injections while titrating the infusion pressure by foot. Since the retinotomy may become enlarged by the cannula tip during the two-step injection process, visualisation of the rise and fall of the bleb on the intraoperative OCT during vector injection helped to indicate that the vector was filling the subretinal space rather than refluxing directly into the vitreous cavity. The sensitivity of OCT also meant foveal detachment could be achieved with shallow subretinal fluid only a

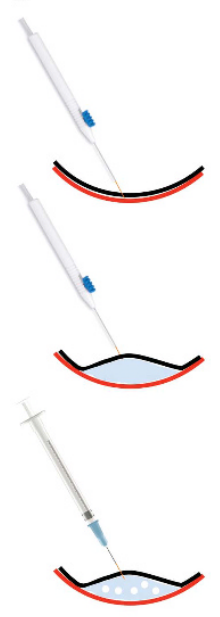

b

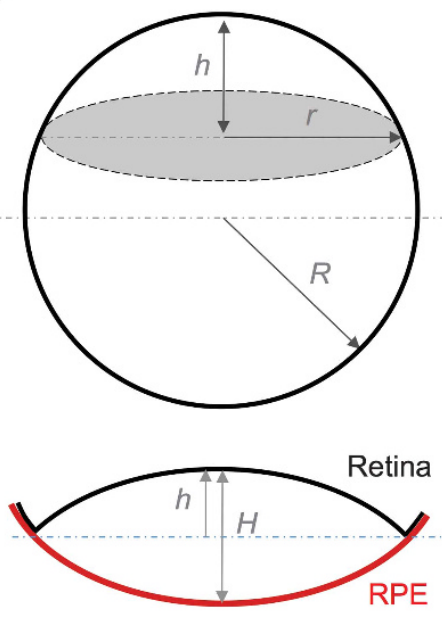

C

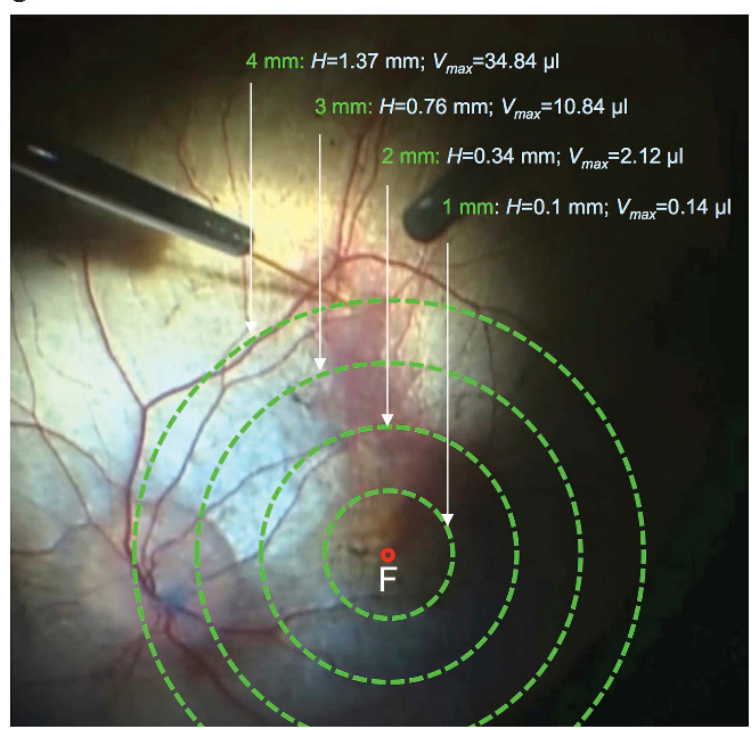

Figure 5 The principle of subretinal bleb formation during gene therapy for choroideremia. (a) A schematic drawing of key surgical steps consisting of the initiation of retinal detachment by subretinal injection of basic saline solution (BSS) using an extendible $41 \mathrm{G}$ cannula (DORC), a necessary step to overcome the abnormally adherent retina of patients with outer retinal degenerations. The $\mathrm{AAV}$ vector solution is then loaded into a $1 \mathrm{ml}$ syringe and slowly infused into the subretinal bleb through the same retinotomy using a dual-bore $41 \mathrm{G}$ cannula (DORC). (b) The spherical cap formula can be used to calculate the height of the subretinal bleb $(H=2 h)$ in relation to the distance of the retinotomy from the fovea $(r)$ and the radius of the whole eye ( $R$, assumed to be half of the average axial length of $24 \mathrm{~mm}$ ). (c) If the retina was assumed to 'flip' from concave to convex without undergoing any elastic stretch during a subretinal injection, for each diameter of subretinal bleb $(r=1,2,3$ or $4 \mathrm{~mm})$, the maximum bleb volume $\left(V_{\max }\right)$ was calculated to be $0.14,2.12,10.84$ and $34.84 \mu \mathrm{l}$, respectively.

(rather than gross foveal elevation visible under the microscope), which minimised foveal stretch.

Furthermore, an intraoperative OCT volume scan taken at the end of vector injection would enable the 
calculation of final bleb volume, giving an indication of the dose given. This has implications for the efficacy of gene therapy, and would be an important consideration when evaluating the outcomes of clinical trials. Since tissue stress would be expected to be highest adjacent to the retinotomy during the induction of the detachment, the initial retinotomy should ideally be placed away from the fovea or areas of retinal thinning, as described previously. ${ }^{10}$ In our experience, a retinotomy located close to the superior vascular arcade at one of the tips of the AF island generally works well in allowing propagation of the detachment towards the fovea. Although $41 \mathrm{G}$ retinotomies are ultimately self-sealing, it may be temporarily leaky especially if enlarged during the injection process, enabling some subretinal fluid to leak out during the recovery period. Since we do not use any air or gas tamponade at the end of the procedure, a superiorly placed retinotomy might allow less vector leakage than an inferior one if the patient postured upright since the vector suspension is denser than BSS.

Another potential source of undesirable retinal stretch during subretinal injection is the presence of air bubbles within the needle or syringes. Sudden forceful expansion of a large air bubble as it emerges from the pressurised injection system into a restricted subretinal bleb could lead to acute retinal stretch and possible macular hole formation, especially in the thin degenerate retina of patients with retinal dystrophies. ${ }^{17,18}$ Stringent efforts were made to purge the BSS and vector syringes of any visible air bubble prior to injection. However, microscopic air bubbles do occasionally appear which were thought have been trapped by the internal ridges at the interphase between the needle shaft and plastic mount. ${ }^{11}$ These microscopic bubbles appeared to be harmless and had a tendency to sit directly under the retinotomy, which was helpful for reducing potential vector reflux during the injection process.

The risk of AAV-induced post-operative vitritis was reduced in a several ways. Perioperative systemic corticosteroid was used to induce transient immunosuppression, which has been well tolerated by all patients treated in several retinal gene therapy trials to date. 2,10,17 The dual-bore design of the subretinal cannula allows egress of vitreous fluid from near the cannula tip to the outside due to the increased intraocular pressure during subretinal injection. This creates a passive suction effect akin to the flute cannula, which may help to remove viral particles refluxing from the retinotomy in the vicinity. At the end of the procedure, the vitreous cavity was irrigated gently to remove any refluxed vector particles.

Irrespective of the therapeutic effects of the gene therapy in the long-term, the retinal structural and functional recovery to baseline seen at one month would suggest that iatrogenic macular detachment can be safely performed as part of the subretinal approach to gene therapy. Future development to make subretinal gene therapy even safer might involve prolonged mechanical infusion of BSS and vector, or a precise one-step OCTguided subretinal infusion of vector. A robotically assisted retinal surgery system to enable 'hands-free' slow subretinal infusion may also achieve these goals. Ultimately developments in gene therapy surgery must go hand-in-hand with improved vector design. It is agreed that a $90 \%$ intravitreal reflux of vector suspension during non-optimal subretinal injection can be compensated by using a vector that is ten times more efficacious. But improving the surgical technique to increase vector delivery from 10 up to $100 \%$ is far easier and more logical.

\section{Summary}

\section{What was known before}

- Subretinal delivery of gene therapy vector would provide targeted treatment of the retinal pigment epithelium (RPE) and photoreceptors in inherited retinal dystrophies.

\section{What this study adds}

- This study shows that iatrogenic macular detachment could be performed as part of the subretinal approach to gene therapy with minimal retinal trauma and describes the surgical technique.

\section{Conflict of interest}

REM: a founding director of NightstaRx (London, UK) - a gene therapy company established by the University of Oxford and funded by the Wellcome Trust. REM is a named inventor on a patent 'Vector administration and dosing' filed on behalf of the University of Oxford (US Patent Application No. 14/598,948). The remaining authors declare no conflict of interest.

\section{Acknowledgements}

We thank Fight for Sight, the Choroideremia Research Foundation and the Hospital Saturday Fund for their generous donations, which helped to fund the OCT microscope. KX is supported by a NIHR clinical lectureship scheme and the University of Oxford. This project is funded by the Efficacy and Mechanism Evaluation (EME) Programme, an MRC and NIHR partnership. The EME Programme is funded by the MRC and NIHR, with contributions from the CSO in Scotland and NISCHR in Wales and the HSC R\&D Division, Public Health Agency in Northern Ireland. 


\section{Disclaimer}

The views expressed in this publication are those of the author(s) and not necessarily those of the MRC, NHS, NIHR or the Department of Health.

\section{References}

1 Jacobson SG, Cideciyan AV, Roman AJ, Sumaroka A, Schwartz SB, Heon E et al. Improvement and decline in vision with gene therapy in childhood blindness. $N$ Engl J Med 2015; 372: 1920-1926.

2 Bainbridge JW, Mehat MS, Sundaram V, Robbie SJ, Barker SE, Ripamonti $C$ et al. Long-term effect of gene therapy on Leber's congenital amaurosis. N Engl J Med 2015; 372: 1887-1897.

3 Edwards TL, Jolly JK, Groppe M, Barnard AR, Cottriall CL, Tolmachova $\mathrm{T}$ et al. Visual Acuity after Retinal Gene Therapy for Choroideremia. N Engl J Med 2016; 374: 1996-1998

4 Bennett J, Wellman J, Marshall KA, McCague S, Ashtari M, DiStefano-Pappas J et al. Safety and durability of effect of contralateral-eye administration of AAV2 gene therapy in patients with childhood-onset blindness caused by RPE65 mutations: a follow-on phase 1 trial. Lancet 2016; 388: 661-672.

5 Ghazi NG, Abboud EB, Nowilaty SR, Alkuraya H, Alhommadi A, Cai $\mathrm{H}$ et al. Treatment of retinitis pigmentosa due to MERTK mutations by ocular subretinal injection of adeno-associated virus gene vector: results of a phase I trial. Hum Genet 2016; 135: 327-343.

6 Feuer WJ, Schiffman JC, Davis JL, Porciatti V, Gonzalez P, Koilkonda RD et al. Gene Therapy for Leber Hereditary Optic Neuropathy: Initial Results. Ophthalmology 2016; 123: 558-570.

7 Rakoczy EP, Lai CM, Magno AL, Wikstrom ME, French MA, Pierce CM et al. Gene therapy with recombinant adenoassociated vectors for neovascular age-related macular degeneration: 1 year follow-up of a phase 1 randomised clinical trial. Lancet 2015; 386: 2395-2403.

8 Maguire AM, High KA, Auricchio A, Wright JF, Pierce EA, Testa F et al. Age-dependent effects of RPE65 gene therapy for Leber's congenital amaurosis: a phase 1 dose-escalation trial. Lancet 2009; 374: 1597-1605.

9 Boye SE, Alexander JJ, Witherspoon CD, Boye SL, Peterson JJ, Clark ME et al. Highly Efficient Delivery of Adeno-Associated Viral Vectors to the Primate Retina. Hum Gene Ther 2016; 27: 580-597.
10 MacLaren RE, Groppe M, Barnard AR, Cottriall CL, Tolmachova T, Seymour L et al. Retinal gene therapy in patients with choroideremia: initial findings from a phase 1/2 clinical trial. Lancet 2014; 383: 1129-1137.

11 Fischer MD, Hickey DG, Singh MS, MacLaren RE. Evaluation of an optimized injection system for retinal gene therapy in human patients. Hum Gene Ther Methods 2016; 27: 150-158.

12 Simunovic MP, Jolly JK, Xue K, Edwards TL, Groppe M, Downes SM et al. The spectrum of CHM gene mutations in choroideremia and their relationship to clinical phenotype. Invest Ophthalmol Vis Sci 2016; 57: 6033-6039.

13 MacLaren RE. Gene therapy for age-related macular degeneration. Lancet 2015; 386: 2369-2370.

14 Xue K, Oldani M, Jolly JK, Edwards TL, Groppe M, Downes SM et al. Correlation of optical coherence tomography and autofluorescence in the outer retina and choroid of patients with choroideremia. Invest Ophthalmol Vis Sci 2016; 57: 3674-3684.

15 Jolly JK, Edwards TL, Moules J, Groppe M, Downes SM, MacLaren RE. A qualitative and quantitative assessment of fundus autofluorescence patterns in patients with choroideremia. Invest Ophthalmol Vis Sci 2016; 57: 4498-4503.

16 Archimedes of Syracuse. On the sphere and cylinder, Archimedes Palimpsest (3rd century BC) http://openn. library.upenn.edu/Data/0014/ArchimedesPalimpsest/.

17 Maguire AM, Simonelli F, Pierce EA, Pugh Jr EN, Mingozzi F, Bennicelli J et al. Safety and efficacy of gene transfer for Leber's congenital amaurosis. N Engl J Med 2008; 358: 2240-2248.

18 Jacobson SG, Cideciyan AV, Ratnakaram R, Heon E, Schwartz SB, Roman AJ et al. Gene therapy for Leber congenital amaurosis caused by RPE65 mutations: safety and efficacy in 15 children and adults followed up to 3 years. Arch Ophthalmol 2012; 130: 9-24.

This work is licensed under a Creative Commons Attribution 4.0 International License. The images or other third party material in this article are included in the article's Creative Commons license, unless indicated otherwise in the credit line; if the material is not included under the Creative Commons license, users will need to obtain permission from the license holder to reproduce the material. To view a copy of this license, visit http://creativecommons.org/licenses/by/ $4.0 /$

(c) The Author(s) 2017

Supplementary Information accompanies this paper on Eye website (http://www.nature.com/eye) 